\section{ISBE - set out for a Systems Biology Infrastructure for Europe}
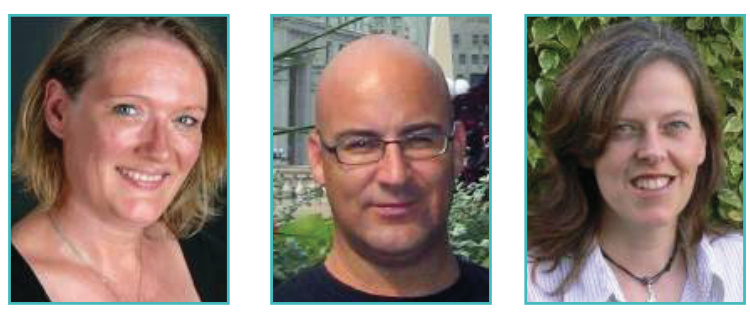

Susanne Hollmann', Vitor AP Martins dos Santos², Babette Regierer ${ }^{2,3}$

'University of Potsdam, Potsdam, Germany

${ }^{2}$ Wageningen University, Wageningen, The Netherlands

${ }^{3}$ LifeGlimmer $\mathrm{GmbH}$, Berlin, Germany

Received 30 June 2013; Published 19 August 2013

Systems biology requires the availability, co-ordination and simultaneous interaction of a large number of diverse facilities and activities. These cover an entire spectrum, from mathematical modelling, through biological, biomedical and clinical experiments, to dedicated technology development. The systems biology community needs close cooperation with data-generation groups and bioinformaticians to define a strategy for producing life-science data of sufficiently high quality for model generation. For each medical, biological or biotechnological problem addressed, the optimal combination of facilities and activities is likely to be different. The complexity of biological systems, and the diversity and dynamics of their processes, means that a full analysis is far too complex to be handled by a single entity, industry or country - a variety of specialist expertise and facilities are typically necessary to achieve results suitable for modelling. Systemslevel approaches for tackling the complexity of life-science data provide a profound conceptual advance compared to reductionist biological research methods of the past. Rather than focusing on individual laboratories, specialising in a limited number of research technologies, the Infrastructure for Systems Biology in Europe (ISBE) will facilitate the synergistic application of a wide range of research techniques and technologies to problems of major medical and biotechnological importance.
The life sciences are both highly diverse and highly fragmented; initiating and supporting community-building activities to create efficient links and mutual cooperation between them is therefore a major issue. A first cross-disciplinary "community building" event in Amsterdam, in November 2012, initiated by the European Coordination Action "AllBio - Broadening the Bioinformatics Infrastructure to Unicellular, Animal and Plant Science ${ }^{l \prime \prime}$, discussed the current challenges in bioinformatics and future needs in the life sciences. Researchers and coordinators of various life science EU initiatives and COST Actions joined the meeting to identify relevant interfaces and mutual problems. As the participants introduced their projects and activities, it became apparent that the communities they represent are still working quite distantly from each other, and it will need a joint effort to establish sufficient communication and interaction between all parties and relevant stakeholders.

To gain a better understanding of the functioning of cells, tissues, organs and whole organisms, it is clear that the biology of the future will necessarily address questions on a large scale using systems biology approaches. This will require the careful building of efficient pipelines, involving data acquisition, analysis, processing, integration, and finally modelling. Utilisation of the information will be the next step towards acquiring and transferring the knowledge gained, for example to clinics, to animal breeding programmes, to pest control in crop plants, and to numerous other new and existing applications. The mathematical and formal modelling of molecular, physiological and anatomical data derived from various biological, biomedical and clinical experiments still present enormous hurdles, and targeted technology development is still needed to close existing analytical gaps.

Based on opportunities offered by national and European funding programs, numerous excellent initiatives and projects have contributed to the current paradigm shift in modern life sciences, preparing the ground for understanding fundamental aspects of life via systems biology. Now it is time to integrate knowledge and expertise across many disparate scientific areas, to create the efficient, cooperative links necessary to amalgamate them successfully.

1 http://www.allbioinformatics.eu 
ISBE is a new initiative to bring such diverse projects together in a single concerted action, to offer their combined wisdom and expertise to Europe's scientific community. Based on the activities of AllBio and other initiatives, ISBE will provide not only a new infrastructure, but also the opportunity to identify links and cross-cutting themes in order both to advance science and to avoid redundancies - it is a unique chance to unite efforts for the benefit of the whole life science community in Europe.

To create a European infrastructure for systems biology, a distributed, but closely interconnected framework will be established that combines data generation, data stewardship (data processing, analysis and curation), and data integration and modelling. Centres across Europe will apply and develop expertise in model-driven knowledge generation, and make this expertise freely available to the community.

The future success of the European economy will depend strongly on our ability to enhance existing and to develop new knowledge-based industries. The ISBE infrastructure will make a considerable impact on European industry, business and society through the systematic study of complex biological processes, incorporating expertise from physics, engineering, biology, chemistry, computer science and mathematics. This will lead to new and important technological applications: for example, in health, agriculture, food production, clean energy, and other areas related to Europe's growing bio-based economy. In particular, the ISBE infrastructure will be highly relevant in transforming our basic knowledge of complex molecular systems into the new arena of predictive, preventive and personalised medicine that reaches across Europe and beyond. The systems biology approaches developed in the ISBE infrastructure will provide new insights and will assist development of tools for designing new medical, biotechnological and environmental applications. ISBE will also transform systems biology into an integrated, pan-European activity by the use of Web-based experimental facilities and on-line mathematical modelling, and make the biological sciences more productive and costeffective. This is important for European society, industry and economy in genera.l

\section{Acknowledgements}

$\underline{\mathrm{ISBE}}^{2}$ is a European ESFRI Infrastructure project comprising 23 partners from 11 countries, and is financially supported by the EC under FP7.

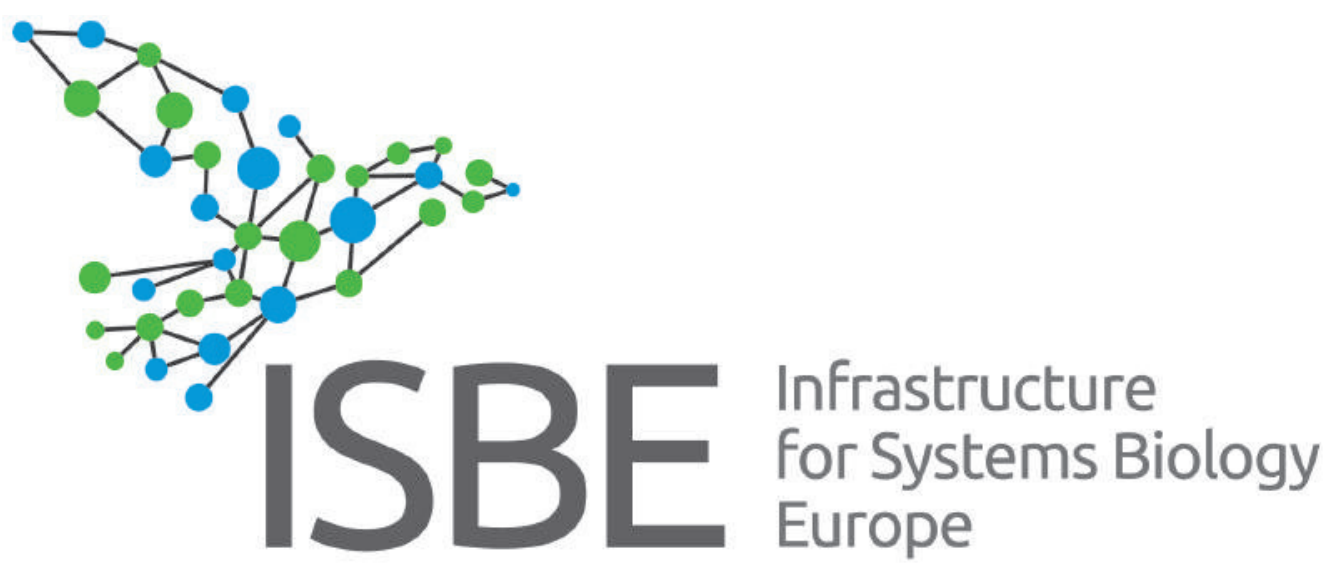

2 www.isbe.eu 